\section{Neue neonatale Risikofaktoren für Asthma bronchiale}

\author{
Asthma ist eine multifaktorielle Erkrankung. So können auch \\ Ereignisse in der Fetal- und Perinatalphase die Entwicklung des \\ Immunsystems und des Respirationstrakts beeinträchtigen. Eine \\ ganze Reihe von Asthmarisikofaktoren in dieser Entwicklungsphase \\ ist schon gesichert, bisher unklar war der Einfluss der neonatalen \\ Phototherapie.
}

$\ln$ einer populationsbasierten Studie wurde zunächst der Datenbestand des Swedish Hospital Discharge Register nach Kindern durchsucht, die zwischen 1987 und 1999 geboren worden waren und die später mit der Diagnose Asthma stationär behandelt wurden. Kinder unter zwei Jahren wurden wegen der unsicheren Asthmadiagnose in diesem Alter ausgeschlossen. Aus dem Datenbestand des Swedish Medical Birth Registry identifizierte man dann alle 1.386.029 Kinder der gleichen Jahrgänge und analysierte durch Datenabgleich mögliche Auffälligkeiten bei den prä- und perinatalen Parametern der später asthmakrank gewordenen Kinder.

Von den zahlreichen in dieser Registerstudie identifizierten Asthmarisikofaktoren waren die meisten bekannt: geringes Alter der Mutter, Rauchen der Mutter während der Schwangerschaft, Zeiten ungewollter Kinderlosigkeit, niedriger Ausbildungsgrad der Mutter, Dia- betes und Präeklampsie der Mutter, Mangelgeburt, Kaiserschnitt und instrumentell assistierte vaginale Entbindung. Einige Erkrankungen beim Neugeborenen erwiesen sich ebenfalls als unabhängige Risikofaktoren: Sepsis oder Pneu-

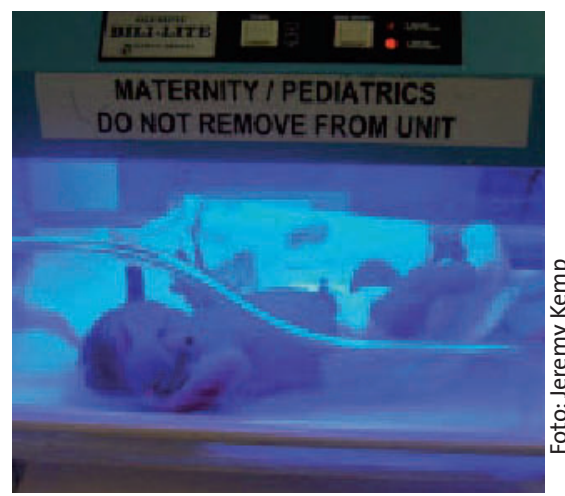

Schwedische Epidemiologen identifizierten einen Neugeborenenikterus und die damit häufig assoziierte Photoherapie als neue Risikofaktoren für Asthma bronchiale. monie, respiratorische Probleme und deren Behandlung sowie Neugeborenenikterus und/oder neonatale Phototherapie. Der letztgenannte Zusammenhang blieb auch nach Ausschluss von Fällen mit anderen Risikofaktoren und nach Adjustierung auf Risikofaktoren bei der Mutter bestehen (Odds Ratio 1,27). Die Assoziation wurde noch deutlicher bei den Kindern, die mehrfach wegen Asthmas eingewiesen wurden (Odds Ratio 1,5). Da die Phototherapie die Standardmaßnahme bei einem Ikterus darstellt, lagen bei fast allen Kindern beide Faktoren vor, so dass sie statistisch nicht getrennt werden konnten.

Über die Ursachen kann anhand der Ergebnisse dieser epidemiologischen Studie nur spekuliert werden. So könnte die Phototherapie das unreife Immunsystem des Neugeborenen beeinflussen und dabei den Shift der zunächst vorliegenden TH2-dominierten Immunantwort in eine TH1-dominierte Immunantwort verlangsamen und so das Asthmarisiko erhöhen.

Fazit: Neonataler Ikterus und/oder eine Phototherapie des Neugeborenen korrelieren mit dem Auftreten von schwerem Asthma später im Leben.

$b k$

Aspberg S et al. Is neonatal phototherapy associated with an increased risk for hospitalized childhood bronchial asthma? Pediatr Allergy Immunol 2007; 18: 313-9

\section{Was ist dran am Plazeboeffekt bei Asthmapatienten?}

\author{
Dass Patienten auf Plazebopräparate reagieren, ist bekannt. Ob \\ diese Effekte nur subjektiver Art oder klinisch objektiv nachweisbar \\ sind, testeten US-amerikanische Forscher in einer Doppelblind- \\ studie an Patienten mit leichtem Asthma bronchiale.
}

W ie objektiv Plazebopräparate wirken können, untersuchten Margaret Kemeney und ihr Team von der Universitiy of California, San Francisco, an 55 Patienten mit leichtem intermittierenden oder persistierenden Asthma bronchiale. Die Studienteilnehmer wiesen alle einen FEV1-Wert von mindes- tens $80 \%$ des Sollwertes auf und wandten keine inhalativen Glukokortikoide oder andere Begleitmedikamente an. Sie waren Nichtraucher; bei keinem von ihnen war in der letzten Zeit eine pulmonale Infektion aufgetreten.

Zunächst unterzogen sich die Patienten im Rahmen der Eingangsuntersu- chung einer Lungenfunktionsprüfung inklusive Methacholin-Provokationstest. Alle Studienteilnehmer mussten nach der Gabe von Salmeterol im Provokationstest einer geringere Hyperreagibilität aufweisen. Im zweiten Teil der Studie erhielten die Patienten etwa eine Woche später im Doppelblindversuch vor dem Methacholintest entweder Plazebo oder Salmeterol. Wieder etwa eine Woche später wurden dann Plazebo und Salmeterol jeweils im Cross-over-Design ausgetauscht. Einem Teil der Patienten gegenüber lobten die Ärzte im Gespräch das eingesetzte Medikament als besonders wirkungsvoll, bei den anderen informierten sie dagegen in neutralem Ton über die Substanz. 